\title{
The chaotic dynamics of jamming
}

\author{
Edward J. Banigan ${ }^{1,2}$, Matthew K. Illich ${ }^{1}$, Derick J. Stace-Naughton ${ }^{1}$ and David A. Egolf ${ }^{1,3 \star}$
}

\begin{abstract}
Granular materials are collections of discrete, macroscopic particles characterized by relatively straightforward interactions. Despite their apparent simplicity, these systems exhibit a number of intriguing phenomena, including the jamming transition, in which a disordered collection of grains becomes rigid when its density exceeds a critical value ${ }^{1,2}$. Many aspects of this transition have been explored, but an explanation of the underlying dynamical mechanisms for the transition remains elusive. Here, applying nonlinear dynamical techniques ${ }^{3-5}$ to simulated two-dimensional Couette shear cells ${ }^{6-8}$, we reveal the mechanisms of jamming and find that they conflict with the prevailing picture of growing cooperative regions. In addition, at the density corresponding to random close packing ${ }^{9,10}$, we find a dynamical transition from chaotic to non-chaotic states accompanied by diverging dynamical length- and timescales. Furthermore, we find that the dominant cooperative dynamical modes are strongly correlated with particle rearrangements and become increasingly unstable before stress jumps, providing a way to predict the times and locations of these striking stress-release events in our simulations.
\end{abstract}

Even after over a century of study, granular materials are not fully understood, probably owing to the unusual importance of dynamical aspects of their behaviour. Interestingly, granular systems at zero temperature undergo a transition from a fluidlike state to a rigid, disordered state known as the jamming transition $^{1,2,11}$ when the granular density is increased. These disordered solids are intriguing (and difficult to understand) owing to the presence of structural heterogeneities, with the internal forces varying strongly with the material ${ }^{2}$. Under weak stress such as slow shear or compression, the behaviour of the disordered, heterogeneous networks of internal forces is fairly static over extended periods of time but is occasionally punctuated by striking, localized rearrangements of the particles.

In recent years, researchers have made progress in understanding the structure of granular systems near the jamming transition by, for example, quantifying force ${ }^{12}$ and contact $^{13}$ networks, measuring quantities such as the dynamic susceptibility ${ }^{13-15}$ and performing critical point scaling analyses ${ }^{16}$. Other work has focused on characterizing the vibrational modes ${ }^{17-19}$, although there is considerable controversy over the relevance of normal mode analysis in the vicinity of the jamming transition ${ }^{19,20}$. This type of analysis has been used well above the jamming density to predict the locations of soft spots where rearrangement events occur ${ }^{18}$. Yet another type of structure-based analysis, applied to a related granular system-a free-standing pile of beads-also shows a small, but measurable, degree of predictive power ${ }^{21}$. We take a different approach and use the mathematics of nonlinear dynamics to reveal and analyse the behaviours of the dominant dynamical modes of a sheared granular system.
Beginning about 15 years ago, a series of seminal experiments on jamming were performed using an annular two-dimensional (2D) Couette shear cell packed with small, bidisperse, birefringent elastic discs at a packing density $\phi$ (refs 6-8). The inner and outer walls of the cell were covered in treads and the inner wall was rotated to shear the material at a constant rate. As $\phi$ was increased over a narrow range, the total pressure in the system increased by many orders of magnitude- the system jammed. The grains formed a network of stress chains ${ }^{6-8}$, and the system exhibited behaviour that was sometimes flowing and sometimes almost static. Measurements of a variety of quantities in experiments ${ }^{6-8}$ and simulations $s^{22,23}$ have provided insight into this transition, but the dynamical mechanisms for the observed behaviours remain unclear.

To elucidate these mechanisms, we perform molecular dynamics simulations of a 2D Couette shear cell and quantify the behaviour using nonlinear dynamical techniques ${ }^{3-5}$. As shown in Fig. 1a, we simulate $N$ soft discs interacting through frictional and dissipative elastic forces ${ }^{22,23}$ in an $L_{x} \times L_{y}$ rectangular cell with periodic boundaries in the $x$-direction, parallel to the counter-moving, treaded, shearing walls. Our system exhibits behaviours qualitatively similar to the corresponding experiments as $\phi$ is varied.

The field of nonlinear dynamics provides tools for untangling the complicated behaviour of a nonlinear system into a hierarchy of spatiotemporal modes, organized by how much each mode drives the dynamics. This hierarchy consists of Lyapunov vectors $\delta \mathbf{u}^{(i)}(t)$ and associated Lyapunov exponents $\lambda_{i}$, indexed by $i=1,2, \ldots$, and ordered by decreasing $\lambda_{i}$. Each Lyapunov vector, $\delta \mathbf{u}^{(i)}(t)$, represents a particular set of time-dependent, infinitesimal, translational perturbations, $\left\{\delta \mathbf{r}_{j}^{(i)}(t)\right\}$, and velocity perturbations, $\left\{\delta \mathbf{v}_{i}^{(i)}(t)\right\}$, for all particles. The first Lyapunov vector, $\delta \mathbf{u}^{(1)}(t)$, is the set of perturbations that grows the fastest (or shrinks the slowest) on average over the evolution of the system - the dominant collective dynamical mode. The first Lyapunov exponent, $\lambda_{1}$, is the average exponential growth rate of $\delta \mathbf{u}^{(1)}(t)$ and can be computed as the average of short-time growth rates $\lambda_{1}^{\Delta t}(t)$ of $\delta \mathbf{u}^{(1)}(t)$ measured over short intervals $\Delta t$ (ref. 5). If $\lambda_{1}>0$, the system is chaotic and thus sensitive to small perturbations. If $\lambda_{1}<0$, any perturbations decay exponentially quickly.

The time-dependent Lyapunov vectors and their short-time growth rates provide detailed information about the heterogeneous dynamics of our system. Figure $1 \mathrm{~b}$ shows the spatial distribution of the magnitude-squared of the first Lyapunov vector, $\left|\delta \mathbf{u}_{j}^{(1)}\left(t_{0}\right)\right|^{2}=\left|\delta \mathbf{r}_{j}^{(1)}\left(t_{0}\right)\right|^{2}+\left|\delta \mathbf{v}_{j}^{(1)}\left(t_{0}\right)\right|^{2}$, for each particle $j$ at a time $t_{0}$. The largest components of the Lyapunov vector (red) are localized to a small region of the cell, and $\left|\delta \mathbf{u}_{j}^{(1)}\left(t_{0}\right)\right|^{2}$ correlates strongly with the square of the velocity of the $j$ th particle, $\left|\mathbf{v}_{j}\left(t_{0}\right)\right|^{2}$, as can be seen by comparing Fig. 1b to Fig. 1c.

\footnotetext{
1Department of Physics, Georgetown University, Washington DC 20057, USA, ${ }^{2}$ Department of Physics and Astronomy, University of Pennsylvania, Philadelphia, Pennsylvania 19104, USA, ${ }^{3}$ Institute for Soft Matter Synthesis and Metrology, Georgetown University, Washington DC 20057, USA `e-mail: egolf@physics.georgetown.edu.
} 

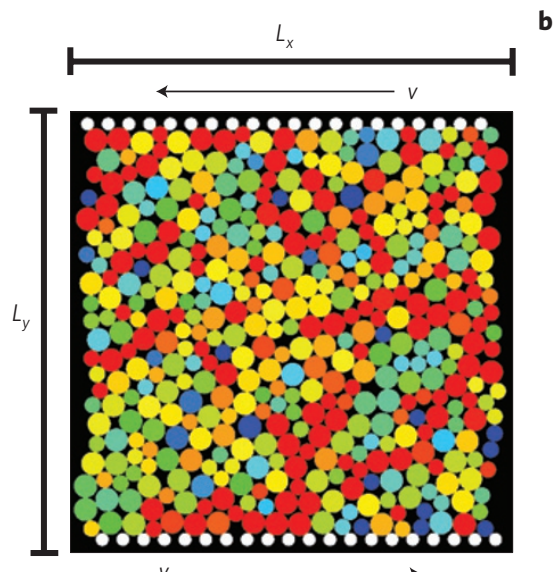

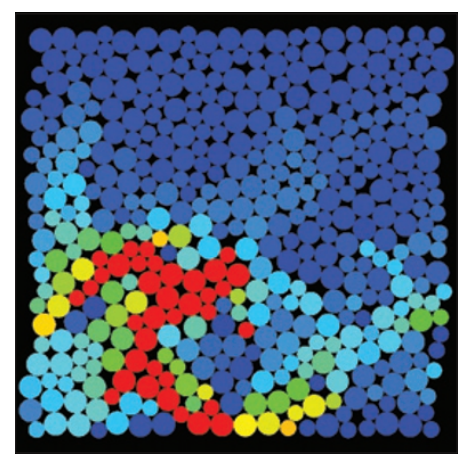

c

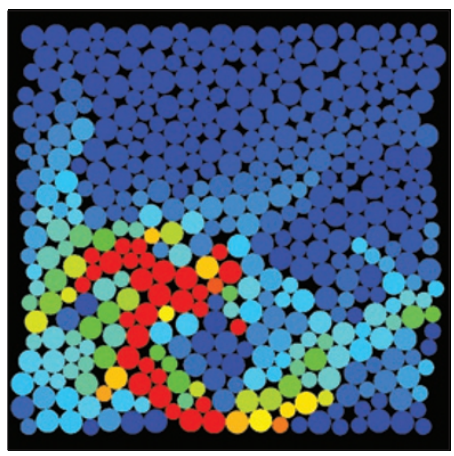

Figure 1 | Visualizations of the stress, Lyapunov vector and velocity fields. Colour-coding denotes the total stress on each disc, from low (blue) to high (red). a, Schematic of an $L_{x} \times L_{y}$ simulated Couette cell with counter-moving treads (white discs) at time to during a stress-release event. The cell is

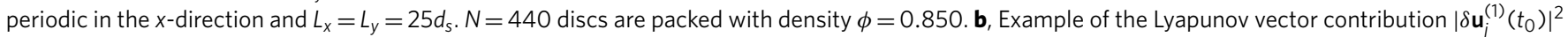
for each disc $j$ at a time $t_{0}$. $\mathbf{c}$, The velocity-squared, $\left|\mathbf{v}_{j}\left(t_{0}\right)\right|^{2}$, for each disc at the same time $t_{0}$.

a

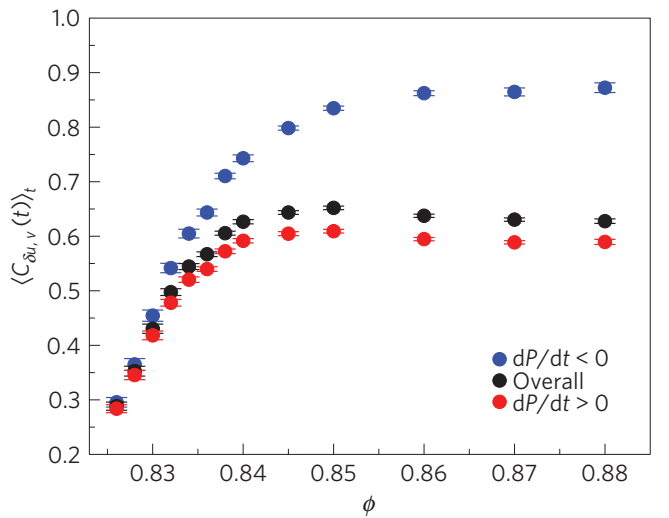

b

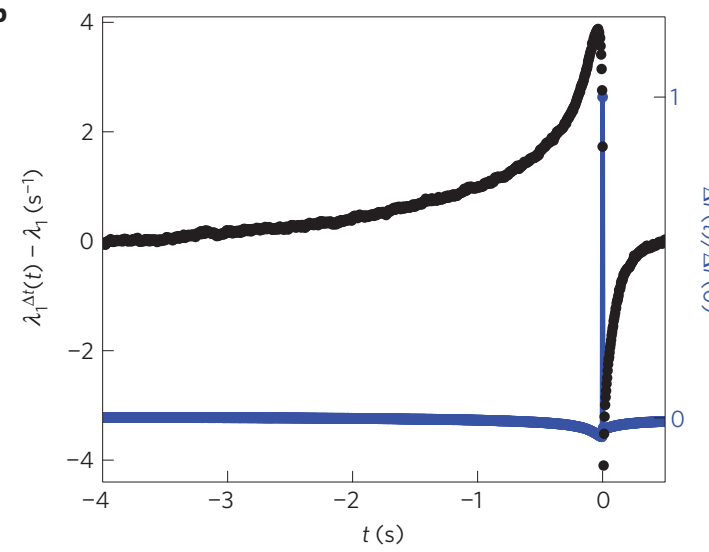

Figure 2 | Correlations between Lyapunov vectors and exponents and system behaviour. a, Time-averaged particle-by-particle cross-correlations between the Lyapunov vector field and velocity field, $\left\langle C_{\delta u, v}^{(1)}(t)\right\rangle_{t}$, as function of packing density $\phi$. Black symbols indicate the averages over all times, whereas blue (red) symbols are the averages over times when the total pressure is decreasing (increasing). All data are for systems of size $L_{x}=L_{y}$ and error bars represent the standard error. b, Blue symbols show the behaviour of pressure changes in the time periods before and after jumps in the pressure larger than 10 standard deviations from the mean. The data are averaged over more than 200,000 of such events. The black symbols show the average behaviour of the short-term growth rates $\lambda_{1}^{\Delta t}(t)$ during the same time periods. The data shown are for $\phi=0.870$ and $L_{x}=4 L_{y}$.
To quantify the correlations between the Lyapunov vectors and the disc velocity fields, we compute the particle-by-particle cross-correlation:

$C_{\delta u, v}^{(1)}(t)$

$=\frac{\sum_{j=1}^{N}\left(\left|\delta \mathbf{u}_{j}^{(1)}(t)\right|^{2}-\left\langle\left|\delta \mathbf{u}_{k}^{(1)}(t)\right|^{2}\right\rangle_{k}\right)\left(\left|\mathbf{v}_{j}(t)\right|^{2}-\left\langle\left|\mathbf{v}_{k}(t)\right|^{2}\right\rangle_{k}\right)}{\sqrt{\sum_{j=1}^{N}\left(\left|\delta \mathbf{u}_{j}^{(1)}(t)\right|^{2}-\left\langle\left|\delta \mathbf{u}_{k}^{(1)}(t)\right|^{2}\right\rangle_{k}\right)^{2}} \sqrt{\sum_{j=1}^{N}\left(\left|\mathbf{v}_{j}(t)\right|^{2}-\left\langle\left|\mathbf{v}_{k}(t)\right|^{2}\right\rangle_{k}\right)^{2}}}$

As can be seen in Fig. 2a (black symbols), the two fields are well correlated, on average. During periods of stress release (blue symbols), the dynamics is dominated by a sequence of rearrangement events and the correlation is remarkably strong; a single dynamical mode typically dominates, at least for dense systems, and $C_{\delta u, v}^{(1)}(t)$ is almost perfect at many times, differing from 1 by less than $0.0001 \%$. (For an example, see Supplementary Fig. S1.) The correlations are higher at larger values of $\phi$ because fewer modes contribute to the dynamics (as quantified by the set of Lyapunov exponents $\left.\left\{\lambda_{i}\right\}\right)$. Altogether, the connection between the fastest moving particles and the most important dynamical modes provides direct evidence that these localized clusters are responsible for cooperative rearrangements. Interestingly, a similar connection was found in supercooled colloidal fluids near the glass transition ${ }^{24}$.

A striking feature of sheared granular systems is the way stress builds in the whole system and is then released through particle rearrangement events in these dynamically unstable regions. These events appear in the time-series of the total pressure $P(t)$ as small jumps in $P(t)$ when the rearranging particles re-jam. The blue line in Fig. 2b shows the average behaviour of $\Delta P(t)$ about a jump at $t=0$. We find that the particles involved in a rearrangement event are in a dynamically unstable configuration for a period of time before the instability is triggered. This is shown by the average behaviour of the short-time growth rate $\lambda_{1}^{\Delta t}(t)$ (black symbols) in the times immediately preceding pressure jumps signalling rearrangement events. For a period of time before the rearrangement, the growth rate becomes positive, indicating instability; this information can be used to predict when these striking slip events are likely and, through the Lyapunov vector, where the events will occur.

To understand the average dynamical properties across the jamming transition, we measure the first Lyapunov exponent, $\lambda_{1}$. As shown in Fig. 3 a for three different packing densities, $\lambda_{1}$ increases with $L_{x}$ (at fixed $L_{y}$ ) but seems to approach an asymptotic value for large system sizes. Figure $3 \mathrm{~b}$ shows that the asymptotic behaviour 
a

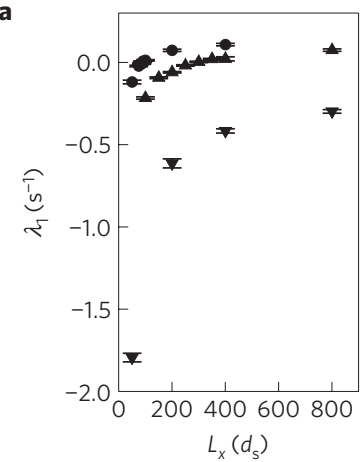

Figure 3 | System size dependence of the Lyapunov exponent. a, Lyapunov exponent $\lambda_{1}$ as a function of system length $L_{x}$ (for fixed $L_{y}=25 d_{s}$ ) for three different packing densities $\phi=0.828$ (circles), $\phi=0.834$ (uptriangles) and $\phi=0.850$ (downtriangles). $\mathbf{b}$, The data in $\mathbf{a}$ are rescaled to show a linear relation between $\lambda_{1}$ and $1 / L_{x}$ for each value of $\phi$. Error bars represent the standard error of the measurements.

of $\lambda_{1}\left(L_{x}\right)$ is consistent with a linear relationship between $\lambda_{1}$ and $1 / L_{x}$ at large $L_{x}$. This relationship seems to hold over a wide range of $\phi$, allowing us to estimate $\lambda_{1}$ as a function of $\phi$ in the thermodynamic limit of infinite-size systems. The filled circles in Fig. 4a show these extrapolated infinite-size $\lambda_{1}$ values as a function of $\phi$. For $\phi<\phi^{*} \approx 0.841$, where $\phi^{*}$ is the jamming density, $\lambda_{1}>0$ so the system is chaotic; for $\phi>\phi^{*}, \lambda_{1}<0$ and the system is no longer chaotic. (This may at first seem counter-intuitive because particles diffuse, but chaos is not required ${ }^{25}$ for diffusion, especially in a driven system.)

Our calculation of $\lambda_{1}$ leads directly to a characteristic dynamical timescale, $\tau_{d}=1 /\left|\lambda_{1}\right|$. The open squares in Fig. 4 a show that as $\phi^{*}$ is approached from below, the dynamics slow markedly as the time it takes for an arbitrary perturbation to grow becomes large; likewise, as $\phi \rightarrow \phi^{*}$ from above, the perturbations decay away over an increasingly long timescale. The transition from a chaotic state to a non-chaotic state occurs for the same range of $\phi$ over which the stress in the system increases by several orders of magnitude-the jamming transition. Moreover, the value of $\phi^{*}$ is consistent with the $2 \mathrm{D}$ random close-packing density ${ }^{9,10}$ that defines the jamming density at zero stress ${ }^{11}$.

Our data suggest that the jamming transition in dynamical systems (or, at least, in our sheared system) is a transition from a chaotic state to a non-chaotic state. This can be viewed as an extension of the idea of rigidity as the defining characteristic of the jammed state ${ }^{11,26}$. Just as a rigid packing is stable to small perturbations, the phase-space trajectory of our jammed, nonchaotic system is also stable (over long times) to perturbations. The zero-stress fluid states are unstable to perturbations, just like our un-jammed, chaotic shear states. In both cases, the important concept is stability; in the zero-stress case, it is stability about a fixed point, whereas in the sheared case, it is stability about a complicated trajectory through phase space.

The inability of the un-jammed states to build significant total pressures seems to be due to the way the unstable events are self-sustaining (on average). Visualizations of the Lyapunov vectors show that the perturbations caused by one rearrangement event propagate, and before they completely dissipate, they often trigger another existing instability in a nearby region. For un-jammed states, the perturbations continue to grow, propagate and trigger other unstable regions. These sequences of events prevent the system from building up significant stresses. For jammed states, the perturbations, on average, die out, so they eventually stop triggering rearrangements, allowing the bulk to build up stress (see, for example, Supplementary Movies S1-S4). Others have studied
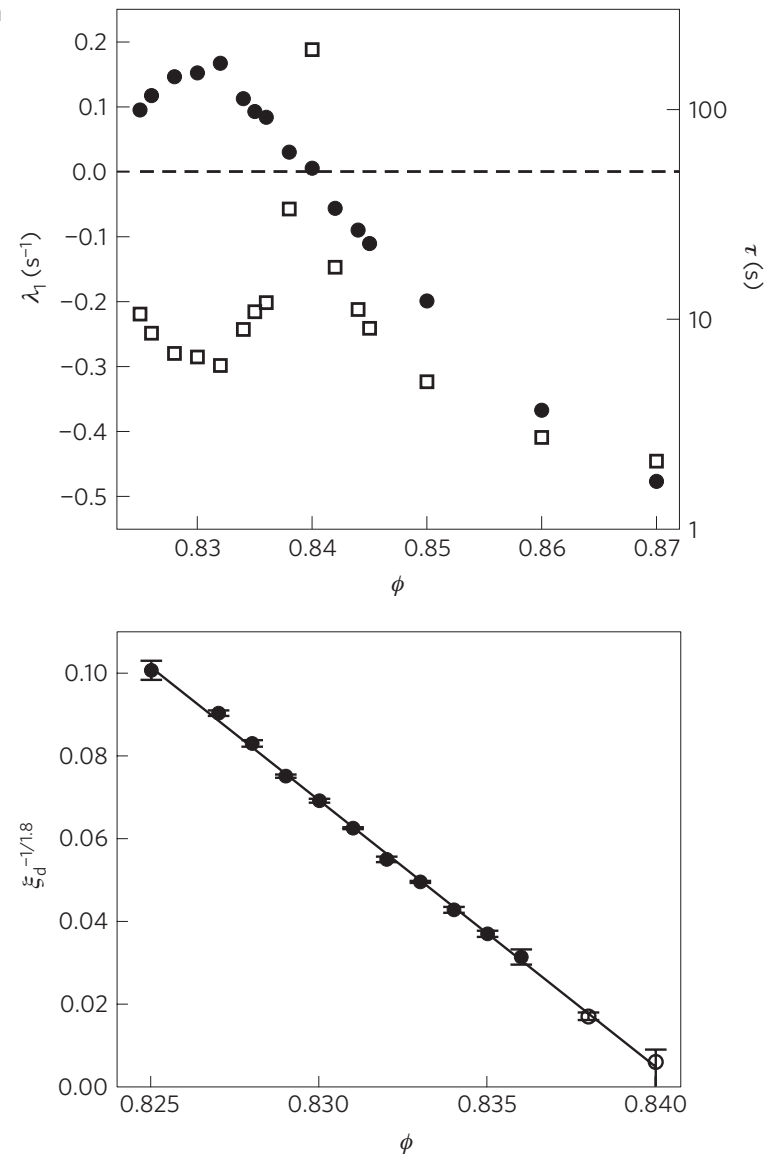

Figure 4 | Time and length scales near the jamming transition. a, Filled circles show the extrapolated infinite system size Lyapunov exponent $\lambda_{1}$ as a function of packing density $\phi . \lambda_{1}$ switches from positive to negative for $0.840<\phi^{*}<0.842$. Open squares show the corresponding dynamical timescales $\tau=1 /\left|\lambda_{1}\right|$. $\mathbf{b}$, Dynamical length scale $\xi_{d}$ (in units of $d_{s}$ ) as a function of packing density $\phi$ approaching the jamming transition from below. Filled circles are interpolated values and open circles are extrapolated values. $\xi_{d}$ is predicted to diverge at $\phi^{*^{\prime}}=0.841$ according to the best-fit power-law $\xi_{d} \propto\left(\phi^{*^{\prime}}-\phi\right)^{-1.8}$ shown as a solid line. The error bars are estimates of the worst-case ranges based on the error bars of the data points used in the interpolations and extrapolations.

correlations in rearrangement events and the propagation of plastic deformations ${ }^{27,28}$ well above the jamming density, and similar studies closer to the jamming transition may shed further light on our findings. Intriguingly, we note that the size of the dynamically cooperative regions remains finite across the jamming transition. The average fraction of the total number of degrees of freedom participating in the most important dynamical mode, as quantified by the average participation ratio $p_{N}=\left\langle 1 /\left[N \sum_{j=1}^{N}\left(\delta x_{j}^{(1)}(t)\right)^{4}+\right.\right.$ $\left.\left.\left(\delta y_{j}^{(1)}(t)\right)^{4}+\left(\delta v_{x_{j}}^{(1)}(t)\right)^{4}+\left(\delta v_{y_{j}}^{(1)}(t)\right)^{4}\right]\right\rangle_{t}$ for a normalized Lyapunov vector $\delta \mathbf{u}^{(1)}(t)$, is almost constant across our entire range of $\phi$ (see Supplementary Fig. S2). Thus, the cooperatively rearranging regions remain a constant size, but the interactions over time of these regions change with $\phi$.

If we now reconsider Fig. 3a in light of our definition of jammed/un-jammed as negative/positive $\lambda_{1}$, we notice that for small values of $L_{x}$ for $\phi=0.828$ (circles) and $\phi=0.834$ (upward triangles), the system is jammed, whereas at larger values of $L_{x}$, the system becomes unjammed. We observe the same behaviour for all values of $\phi \leq 0.836$. Computational limitations prevent us from simulating large enough systems for long enough times to observe this effect directly for $\phi>0.836$, but extrapolations of 
$\lambda_{1}\left(L_{x}\right)$ indicate that the effect will continue up to $\phi \approx \phi^{*}$. This change in the dynamics suggests a definition of a characteristic dynamical length scale, $\xi_{d}$, as the smallest system length $L_{x}$ for which the dynamics are chaotic. This definition of a dynamical length scale is an approximation of the chaotic length scale, $\xi_{f}$, introduced in ref. 4 to characterize the spatial extent of one active dynamical degree of freedom.

We calculate $\xi_{d}$ by measuring $\lambda_{1}$ for a variety of lengths $L_{x}$ and either interpolating between data points on either side of $\lambda_{1}=0(0.825 \leq \phi \leq 0.836)$ or extrapolating $\lambda_{1}\left(L_{x}\right)$ $(0.836<\phi \leq 0.840)$. We find that $\xi_{d}$ gets large as $\phi$ approaches the jamming transition from below. The diverging length scale is well-fitted by a power law of the form $\xi_{d} \sim\left(\phi^{*^{\prime}}-\phi\right)^{-\alpha}$ with $1.7<\alpha<2.1$, and the best fit, with $\alpha=1.8$ and $\phi^{*^{\prime}}=0.841$, is shown in Fig. $4 \mathrm{~b}$. This value of $\phi^{*^{\prime}}$ is once again consistent with the zero-stress transition at the $2 \mathrm{D}$ random close-packing density ${ }^{11}$.

Using widely applicable nonlinear dynamical techniques, we have uncovered the dominant cooperative modes in jamming and found that they can be used to predict the times and locations of rearrangement events in our simulations. Intriguingly, rather than growing in spatial extent, these modes are associated with a diverging dynamical length scale arising from the interplay of the density of unstable regions and the spread of disturbances. In addition, our proposal that jamming in sheared systems is defined by a transition from a chaotic state to a non-chaotic state provides a rigorous and consistent definition for jamming away from the zero-stress limit, which, until now, has been lacking ${ }^{2}$.

\section{Methods}

We simulate an $L_{x} \times L_{y}$ shear cell populated with a 50:50 binary mixture of $N$ soft discs at a packing density $\phi=A_{d} / A_{c}$, where $A_{d}$ is the area covered by the discs and $A_{c}$ is the total area of the cell. The discs have a diameter ratio of 1.4:1 with the smaller discs having a diameter of $d_{s}=7 \mathrm{~mm}$. To achieve arbitrary values of $\phi$ while maintaining a 50:50 population, the diameter ratio is adjusted by less than $1 \%$. The cell is periodic in the $x$-direction, with the shearing walls moving at $\pm 0.1 \mathrm{~mm} \mathrm{~s}^{-1}$ and studded with semi-discs of diameter $0.8 d_{s}$ with centres $1.25 d_{s}$ apart. For all runs, $L_{y}=25 d_{s}$, but $L_{x}$ is varied. The phase space is composed of the $2 N$ components of the position vectors, $\left\{\mathbf{r}_{j}\right\}$, of the $N$ discs indexed by $j$, and the $2 N$ components of the velocity vectors, $\left\{\mathbf{v}_{j}\right\}$, quantifying the deviation from a linear shear profile. We model the interactions of the discs with each other and with the walls using Hookean forces, viscous normal dissipation and frictional forces ${ }^{22,23}$. The discs slide on a frictionless surface. Other material and interaction parameters ${ }^{22,23}$ are designed to match experimental conditions ${ }^{6-8}$

We note that the transition points $\phi^{*}$ and $\phi^{*^{\prime}}$ are determined using discs that are frictional in a system with a small, but finite, shear rate. Using our wall velocities and a characteristic time based on the repulsive forces, we determine our characteristic shear rate to be $\dot{\gamma} \approx 10^{-6}$. Careful scaling studies in ref. 16 indicate that, at this shear rate, the jamming density should be within about 0.001 or 0.002 of the no-shear jamming transition point. Frictional forces between particles can markedly lower the jamming transition point ${ }^{29}$; however, we expect that those effects are small in our system because of the form of our frictional forces. To avoid a discontinuity at zero relative tangential velocity $\mathbf{v}_{i j}^{(t)}$, we employ a frictional force that is proportional to $\mathbf{v}_{i j}^{(t)}$ when $\mathbf{v}_{i j}^{(t)}$ is small and then switches to Coulombic friction at larger velocities ${ }^{22,23}$. Owing to our slow shear rate, most velocities are small, except during rearrangement events, and so our effective friction coefficient is quite small, especially during nearly static times. We expect that the jamming point will not be affected by this friction to within the precision of our measurements.

As we know the equations describing the evolution of our $N$-disc system, we can perform a standard derivation of the tangent space ${ }^{4,5}$ to determine the equations for the time-dependent Lyapunov vectors (and corresponding Lyapunov exponents) describing the geometry of the attractor in its phase space. This process avoids the problem of finding recurrences in a high-dimensional phase space, as would be necessary if we had only observed data. The equation for each Lyapunov vector, $\delta \mathbf{u}^{(i)}(t)$, describes the evolution of a particular set of infinitesimal translational perturbations, $\left\{\delta \mathbf{r}_{j}^{(i)}\right\}$, and infinitesimal velocity perturbations, $\left\{\delta \mathbf{v}_{i}^{(i)}\right\}$, for all particles. The first Lyapunov vector is the set of evolving perturbations that will grow the fastest (or shrink the slowest) over infinite time. We note that these are true dynamical quantities- the first Lyapunov vector at time $t$ does not represent the most unstable mode at that moment; it is the time-dependent state that is the most unstable mode of the long-time evolution of the entire system through its phase space.
Even though there are $4 \mathrm{~N}$ Lyapunov vectors describing the structure of the attractor in phase space, the first Lyapunov vector is easily found by evolving a random set of perturbations. As the first Lyapunov vector grows exponentially faster (or shrinks exponentially slower) than any other Lyapunov vector, the component of the initial random perturbations aligned with the first vector will dominate after a short time. The second Lyapunov vector can be found by evolving another set of perturbations and projecting out the first vector, and so on. The Lyapunov exponents are the growth rates of the Lyapunov vectors averaged over long times.

We integrate the equations for the Lyapunov vectors and the equations for the underlying $N$-disc system using a fifth-order Gear predictor-corrector algorithm ${ }^{30}$ with a time step of $\Delta t=0.00004 \mathrm{~s}$. The discs are initially placed in a random, non-overlapping pattern in a cell $L_{x} \times 3 L_{y}$ and then the walls are slowly moved closer until the cell is $L_{x} \times L_{y}$. After that, we typically wait $200 \mathrm{~s}$ before recording data for $400 \mathrm{~s}$. The Lyapunov vectors $\delta \mathbf{u}^{(i)}(t)$ are renormalized $\left(\sum_{j}\left|\delta \mathbf{u}_{j}^{(i)}\right|^{2}=1\right)$ and short-time growth rates $\lambda_{i}^{\Delta t}(t)$ are calculated at each time step. To isolate the dynamics of the particle network, particles with 0 or 1 disc or wall interactions and particles with 2 interactions that are part of a chain with a free end are excluded from the Lyapunov vectors during that time step, and growth rate calculations are adjusted accordingly. To obtain the desired level of accuracy for measurements of quantities such as Lyapunov exponents, averages are calculated using several hundred runs of different initial conditions.

\section{Received 2 November 2012; accepted 25 February 2013; published online 24 March 2013}

\section{References}

1. Liu, A. J. \& Nagel, S. R. Jamming is not just cool anymore. Nature 396, 21-22 (1998).

2. Liu, A. J. \& Nagel, S. R. The jamming transition and the marginally jammed solid. Annu. Rev. Condens. Matter Phys. 1, 347-369 (2010).

3. Eckmann, J. P. \& Ruelle, D. Ergodic theory of chaos and strange attractors. Rev. Mod. Phys. 57, 617-656 (1985).

4. Cross, M. C. \& Hohenberg, P. C. Pattern formation outside of equilibrium. Rev. Mod. Phys. 65, 851-1112 (1993).

5. Ott, E. Chaos in Dynamical Systems (Cambridge Univ. Press, 1993).

6. Howell, D., Behringer, R. P. \& Veje, C. Stress fluctuations in a 2D granular Couette experiment: A continuous transition. Phys. Rev. Lett. 82, 5241-5244 (1999).

7. Veje, C. T., Howell, D. W. \& Behringer, R. P. Kinematics of a two-dimensional granular Couette experiment at the transition to shearing. Phys. Rev. E 59, 739-745 (1999).

8. Bi, D., Chakraborty, B. \& Behringer, R. P. Jamming by shear. Nature 480, 355-358 (2011).

9. Bideau, D. \& Troadec, J. P. Compacity and mean coordination number of dense packings of hard discs. J. Phys. C 17, L731-L735 (1984).

10. O'Hern, C. S., Langer, S. A., Liu, A. J. \& Nagel, S. R. Random packings of frictionless particles. Phys. Rev. Lett. 88, 075507 (2002).

11. O’Hern, C. S., Silbert, L. E., Liu, A. J. \& Nagel, S. R. Jamming at zero temperature and zero applied stress: The epitome of disorder. Phys. Rev. E 68, 011306 (2003)

12. Kondic, L. et al. Topology of force networks in compressed granular media. Europhys. Lett. 97, 54001 (2012).

13. Coulais, C., Behringer, R. \& Dauchot, O. Dynamics of the contacts reveals Widom lines for jamming. Europhys. Lett. 100, 44005 (2012).

14. Lechenault, F., Dauchot, O., Biroli, G. \& Bouchaud, J. Critical scaling and heterogeneous superdiffusion across the jamming/rigidity transition of a granular gas. Europhys. Lett. 83, 46003 (2008).

15. Heussinger, C., Berthier, L. \& Barrat, J-L. Superdiffusive, heterogeneous, and collective particle motion near the fluid-solid transition in a thermal disordered materials. Europhys. Lett. 90, 20005 (2010).

16. Olsson, P. \& Teitel, S. Critical scaling of shearing rheology at the jamming transition of soft-core frictionless disks. Phys. Rev. E 83, 030302(R) (2011).

17. Xu, N., Vitelli, V., Liu, A. J. \& Nagel, S. R. Anharmonic and quasi-localized vibrations in jammed solids—modes for mechanical failure. Europhys. Lett. 90, 56001 (2010).

18. Manning, M. L. \& Liu, A. J. Vibrational modes identify soft spots in a sheared disordered packing. Phys. Rev. Lett. 107, 108302 (2011).

19. Ikeda, A., Berthier, L. \& Biroli, G. Dynamic criticality at the jamming transition. J. Chem. Phys. 138, 12A507 (2013).

20. Schreck, C., Bertrand, T., O’Hern, C. \& Shattuck, M. Repulsive contact interactions make jammed particulate systems inherently nonharmonic. Phys. Rev. Lett. 107, 078301 (2011).

21. Ramos, O., Altshuler, E. \& Måløy, K. Avalanche prediction in a self-organized pile of beads. Phys. Rev. Lett. 102, 078701 (2009).

22. Schöllmann, S. Simulation of a two-dimensional shear cell. Phys. Rev. E 59, 889-899 (1999). 
23. Lätzel, M., Luding, S. \& Herrmann, H. J. Macroscopic material properties from quasi-static, microscopic simulations of a two-dimensional shear-cell. Granul. Matter 2, 123-135 (2000).

24. Weeks, E. R., Crocker, J. C., Levitt, A. C., Schofield, A. \& Weitz, D. A. Three-dimensional direct imaging of structural relaxation near the colloidal glass transition. Science 287, 627-631 (2000).

25. Cecconi, F., Cencini, M. \& Vulpiani, A. Transport properties of chaotic and non-chaotic many particle systems. J. Stat. Mech.: Theor. Exp. P12001 (2007).

26. Wyart, M., Nagel, S. R. \& Witten, T. A. Geometric origin of excess low-frequency vibrational modes in weakly connected amorphous solids. Europhys. Lett. 72, 486-492 (2005).

27. Lemaître, A. \& Caroli, C. Rate-dependent avalanche size in athermally sheared amorphous solids. Phys. Rev. Lett. 103, 065501 (2009).

28. Lerner, E. \& Procaccia, I. Locality and nonlocality in electroplastic responses of amorphous solids. Phys. Rev. E 79, 066109 (2009).

29. Silbert, L. Jamming of frictional sphere and random loose packing. Soft Matter 6, 2918-2924 (2010)

30. Allen, M. P. \& Tildesley, D. J. Computer Simulation of Liquids (Oxford Univ. Press, 1989).

\section{Acknowledgements}

We thank D. Blair, C. Goodrich, J. Kerin, A. Liu and J. Urbach for discussions. Computational resources were provided on the University Information Services High Performance Computing Shared Cluster Matrix at Georgetown University. This work was supported by the US National Science Foundation (DMR-0094178) and Research Corporation. D.A.E. also received support from the Alfred P. Sloan Foundation. E.J.B. received further support from NSF DMR-1104637 and Penn MRSEC NSF DMR-1120901.

\section{Author contributions}

All authors contributed extensively to the work presented in this paper. E.J.B. and D.A.E. prepared the manuscript.

\section{Additional information}

Supplementary information is available in the online version of the paper. Reprints and permissions information is available online at www.nature.com/reprints. Correspondence and requests for materials should be addressed to D.A.E.

\section{Competing financial interests}

The authors declare no competing financial interests. 